\title{
Biological Factors Influencing the Mice Forced Swim Test
}

\author{
Cong-Cong $\mathrm{Qi}^{1}{ }^{12}, \mathrm{Yu}-\mathrm{Qiang}$ Ding ${ }^{1}$, and Jiang-Ning Zhou ${ }^{2 *}$
}

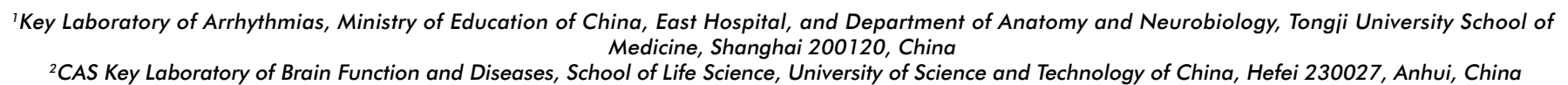

Article Info

\section{Article Notes}

Received: June 02, 2016

Accepted: July 21, 2016

\section{${ }^{*}$ Correspondence:}

Dr. Jiang-Ning Zhou

School of Life Science, University of Science and Technology of China, Huangshan Road 443, Hefei 230027, Anhui, China.

Telephone: 008655163607658 ,

Fax: 008655163607778 ,

Email: jnzhou@ustc.edu.cn

() 2016 Zhou JN. This article is distributed under the terms of the Creative Commons Attribution 4.0 International License

\section{Keywords}

Mice

Forced swim test

Strain

Gender

Age

Susceptibility

\section{ABSTRACT}

The forced swim test (FST), originally developed by Porsolt et al., is highly valuable for assessing the antidepressant-like effects of the majority of currently-available antidepressants. Lucki et al. modified some parameters of the traditional FST in order to facilitate the differentiation between serotonergic and noradrenergic classes of antidepressant drugs. In addition, the FST is one of the most commonly used models for assessing antidepressantlike behaviors in both rats and mice. Focus on the present neuroscience field, knockout and transgenic mice provide a tool for assessing the mechanisms of action of antidepressants, and the factors influencing these behavior in the FST should be taken into considerations. In this MiniReview, we reviewed several biological factors (e.g. strain, gender, age, susceptibility) that may influence mice behavior in the FST and attempt to describe those variables that should be considered when designing studies employing the FST.

\section{Introduction}

Major depressive disorder (MDD) affects one out of every five people in their lifetime and is the leading cause of disability worldwide $^{1}$. Nevertheless, mechanisms associated with the pathogenesis of MDD have yet to be completely understood, and current treatments remain ineffective in a large subset of patients ${ }^{2}$. Therefore, a major emphasis in modern psychiatric research is to uncover the underlying aetiology of depression. A key component of the research is the use of animal models, which are predictive of antidepressant activity ${ }^{3}$. The forced swim test (FST) is one of the most widely used tests across laboratories for assessing symptoms of depression. The protocol takes place over a 48-h period, and is followed by video analysis of the test. The swim test involves the scoring of active (swimming and climbing) or passive (immobility) behavior when rodents are forced to swim in a cylinder from which there is no escape. For both the traditional and modified FST versions, a pre-test of $15-\mathrm{min}$ or 10 - $\mathrm{min}$ is included, as this accentuates the different behaviors in the 5-min swim test following drug treatment ${ }^{4-6}$. Reduction in passive behavior is interpreted as an antidepressant-like effect of the pharmacological intervention provided it does not increase general locomotor activity, which could provide a false positive result in the $\mathrm{FST}^{3}$.

Over the years, researchers have made modifications to the FST to enhance its sensitivity, specificity and reliability ${ }^{7,8}$. The advantages of the FST consist of its ease of use, reliability across different laboratories and trials and the ability to detect a broad spectrum of antidepressants ${ }^{9}$. However, the acute effects of antidepressants may 
also reflect increased arousal, and behavioural changes observed from the first to the second FST exposure could also mirror cognitive processes and interindividual differences in stress coping ${ }^{10-12}$. In general, many biological factors affect behavioral performance during the FST and might influence the evaluation of potential antidepressant drugs. For mice, these factors include strain ${ }^{13,14}$, gender $^{15}$, age $^{16}$, and susceptibility in swimming ${ }^{17}$.

\section{Strain}

Strain is one of the most important parameters to consider in the FST $^{14}$. The influence of the strain of rodent on behavior in the FST has been more extensively characterized in mice than rats, where more than a tenfold difference in baseline immobility has been observed between different strains, including inbred strains (BALB/ cJ, DBA/2J, C57BL/6J, A/J, FVB/NJ, C3H/HeJ, 129/SvemJ, etc.) and outbred strains (Swiss-Webster, CD-1, CF-1, NIH Swiss, NMRI, etc.) $)^{3,14,18 .}$

In general, inbred strains demonstrated lower variability than outbred strains ${ }^{14}$. Different strains showed different baseline immobility and swimming. BALB/cJ and C57BL/6J inbred male mice had lower immobility than the NMRI outbred male mice, and C57BL/6J mice had the highest swimming distance among the three strains $^{19}$. Moreover, the C57BL/6J mice had almost highest immobility among the inbred strains, and more reliable than the others ${ }^{13,14,19}$. This might explain why so many depression-related transgenic mice were based on the genome of C57BL/6J mouse strain ${ }^{20,21}$.

Mice strains not only differ in baseline activity, but also differ in responsiveness to drugs in the $\mathrm{FST}^{8}$. Lucki et al. demonstrated that the DBA/2J and C57BL/6J inbred mice showed greater sensitivity than other strains to desipramine. Nicotine increased swim distance in C57BL/6J and BALB/cJ mice, but did not affect NMRI mice $^{19}$. Different antidepressant drugs probably exert their effects in the FST through partly different mechanisms. On the other hand, outbred mice strains are more responsive to antidepressants in the FST than inbred strains ${ }^{8}$. The most frequently used out strains, CD-122, NMRI ${ }^{19}$ and Swiss $^{23}$, respond positively to most of the antidepressants when subjected to the FST $^{8}$. The strain background is a critical variable in determining baseline performance and the sensitivity to different types of antidepressant drugs in the mouse FST.

\section{Gender}

Behavioral strategies in the FST can vary significantly with animal gender. Firstly, gender-specific immobility differences were shown to exist in many mice strains. For example, male WT mice showed significantly less immobility during the night phase in comparison to female mice ${ }^{24}$. Secondly, gender-specific immobility in FST was also existed in genotype mice. Lydia et al. found that early-life interventions were able to improve the time and frequency of episodes of immobility, being more evident in the female gender of both old NTg and 3xTg-Alzheimer's disease mice ${ }^{16}$. In addition, there were differences in the circadian characteristics of immobility induced by FST in WT, Clock ${ }^{\Delta 19}$, Per1, and Per2 mutant mice, and all four genotypes showed gender-specific differences in the level of immobility ${ }^{24}$.

C57BL/6J mice are commonly used as the genetic background for many knockout and transgenic mice lines ${ }^{12,15,20,25}$, and studies found female C57BL/6J mice to be more sensitivity to antidepressants in the FST. Females C57BL/6J naïve mice tended to show a greater response to antidepressant amitriptyline treatment than males, and female stress-naïve mice are more sensitive to the rapid and the sustained (at 24h) antidepressant-like effects of ketamine, which may be due to altered sensitivity to the drug or variations in metabolism or consumption ${ }^{15,26}$. Moreover, The residual (i.e., after withdrawal) antidepressant effects mediated by PAM-2 or N,6-dimethyltricyclo[5.2.1.0 $0^{2,6}$ ] decan-2-amine enantiomers were only observed in female $\operatorname{mice}^{27,28}$.

Age

Evidence suggested that there was a strong difference between younger and older mice groups in the FST. Older (8 to 12 month old) naïve male C57BL/6J mice had lower immobility than their younger ( 2 to 3 month old) counterparts $^{29}$. There was also a significant difference in immobility between 22-month-old mice, 17-month-old mice and 11-month-old mice ${ }^{30}$. Shortly, older C57BL/6J mice had decreased immobility. Moreover, transgenic mice also showed age-depended behavioral performance in the FST. The long persistence of immobility found in males 17-month-old (late-stages of disease) 3xTg-AD mice was different to that at 12 months of age (beginning of advanced stages $)^{16}$.

Sensitivity to some antidepressants is also profoundly altered by the age of mice. Tricyclics, noradrenaline reuptake inhibitors and serotonin reuptake inhibitors exhibited stronger effects in 4-week-old Swiss mice than 40 -week-old mice ${ }^{8,31}$. Social isolation, as well as treatment with reserpine, an antihypertensive and antipsychotic drug, increased FST immobility in 17-21 day-old SwissWebster mice but not in 26-30-day-old mice ${ }^{32,33}$.

\section{Mice Susceptibility in Swimming}

It was recently found that the mice susceptibility in swimming proficiency is another important factor influencing the mice FST. It has been reported that male mice that are genetically selected for long attack latency 
(LAL) and short attack latency (SAL) display differences in the structural and functional properties of postsynaptic serotonergic-1A (5-HT 1A) receptors ${ }^{34}$ and that they also show divergent behavioral responses during the FST, such as higher immobility in LAL and lower immobility in SAL mice ${ }^{35}$. Moreover, our group found that individual ICR mouse performed differently in the forced swim pretest, resulting in two different mouse substrains: short immobility mice (SIM) and long immobility mice (LIM), and the SIM substrain showed a greater susceptibility to forced swimming. In other words, leaned helplessness is present in the SIM substrain but not the LIM substrain in our experimental conditions.

Sensitivity to some antidepressants is also profoundly altered by the mice susceptibility in swimming. Acute administration of the full 5-HT $1 \mathrm{~A}$ receptor agonist, 8-OH-DPAT $(5 \mathrm{mg} / \mathrm{kg})$, induced a significant decrease in immobility and an increase in swimming activity in LAL mice, but it did not affect them in SAL mice ${ }^{34}$. Our study also showed that short-term abscisic acid administration had antidepressant-like effects only in the SIM substrain, as indicated by decreased immobility and increased swimming in the FST. This result highlighted the importance of the forced swimming pre-test, which is used to screen out the SIM substrain in preclinical antidepressants studies. Along with the positive correlation of pre-test performance with FST in immobility, these results suggest that mice sensitivity to forced swimming was positively correlated with drug sensitivity, further confirming that mice substrains grouping is necessary ${ }^{17}$. These results also suggest that susceptibility in swimming is strongly related to mouse genetic background.

\section{Conclusion}

Mice sensitivity to the FST can be influenced by many factors including: preconditioning before the FST, schedule and routes of treatment, dosage and type of the drugs as well as experimental design and laboratory environmental effects $^{8}$. In the present Mini Review, we analysed the variables of biological factors: strain, gender, age, and susceptibility, and highlighted their importance in the FST and antidepressants screening.

The detailed characterization of strain, gender, age, and susceptibility related changes in behavior of mice will provide researchers with useful information for designing behavioral experiments, interpreting results, and understanding the neurobiological basis of related behavioral changes ${ }^{29}$. Especially, our previous results suggest that dividing ICR mice into drug-sensitive and druginsensitive groups may not only allow easier screening for anti-depressant drugs, but also provide opportunities for identifying drugs that are effective in patients with drugresistant depression if drugs can be developed that are also effective in the LIM group. Many factors affect behavioral performance during the FST and might influence the evaluation of potential antidepressant drugs ${ }^{17}$. In this Mini Review, sensitivity to the antidepressants is profoundly altered by all the biological factors, which suggested that neuropharmacological mechanism of drug action is critical for the interpretation of FST results ${ }^{36}$. Antidepressant drug belongs to diverse chemical class with different acute pharmacological effect and mechanisms of action ${ }^{37}$, and the type and dose of drug as another important factor of mice FST should be considered. Due to the high sensitivity of the test to biological variability, the FST should be standardized and the above-mentioned factors should be considered during study design and execution.

\section{Acknowledgements}

This work was supported by the Postdoctoral Science Foundation of China (2016M591714).

\section{References}

1. Kessler RC, Chiu WT, Demler O, Merikangas KR, Walters EE. Prevalence, severity, and comorbidity of 12-month DSM-IV disorders in the National Comorbidity Survey Replication. Arch Gen Psychiatry. 2005;62(6):617-627.

2. Menard C, Hodes GE, Russo SJ. Pathogenesis of depression: Insights from human and rodent studies. Neuroscience. 2016;321:138-162.

3. Slattery DA, Cryan JF. Using the rat forced swim test to assess antidepressant-like activity in rodents. Nature Protocols. 2012;7(6):1009-1014.

4. Porsolt RD, Le Pichon M, Jalfre M. Depression: a new animal model sensitive to antidepressant treatments. Nature. 1977;266(5604):730732.

5. Lucki I. The forced swimming test as a model for core and component behavioral effects of antidepressant drugs. Behavioural pharmacology. 1997;8(6-7):523-532.

6. Detke MJ, Lucki I. Detection of serotonergic and noradrenergic antidepressants in the rat forced swimming test: the effects of water depth. Behavioural brain research. 1996;73(1-2):43-46.

7. Chen L, Faas GC, Ferando I, Mody I. Novel insights into the behavioral analysis of mice subjected to the forced-swim test. Translational psychiatry. 2015;5:e551.

8. Petit-Demouliere B, Chenu F, Bourin M. Forced swimming test in mice: a review of antidepressant activity. Psychopharmacology. 2005;177(3):245-255.

9. Borsini F, Meli A. Is the forced swimming test a suitable model for revealing antidepressant activity? Psychopharmacology (Berl). 1988;94(2):147-160.

10. Taghzouti $K$, Lamarque $S$, Kharouby $M$, Simon $H$. Interindividual differences in active and passive behaviors in the forced-swimming test: implications for animal models of psychopathology. Biol Psychiatry. 1999;45(6):750-758.

11.West AP. Neurobehavioral studies of forced swimming: the role of learning and memory in the forced swim test. Prog Neuropsychopharmacol Biol Psychiatry. 1990;14(6):863-877.

12. Bachli H, Steiner MA, Habersetzer U, Wotjak CT. Increased water temperature renders single-housed C57BL/6J mice susceptible to antidepressant treatment in the forced swim test. Behavioural brain research. 2008;187(1):67-71. 
13. Crowley JJ, Blendy JA, Lucki I. Strain-dependent antidepressantlike effects of citalopram in the mouse tail suspension test. Psychopharmacology (Berl). 2005;183(2):257-264.

14. Lucki I, Dalvi A, Mayorga AJ. Sensitivity to the effects of pharmacologically selective antidepressants in different strains of mice. Psychopharmacology (Berl). 2001;155(3):315-322.

15. Caldarone BJ, Karthigeyan K, Harrist A, Hunsberger JG, Wittmack E, King SL, et al. Sex differences in response to oral amitriptyline in three animal models of depression in C57BL/6J mice. Psychopharmacology (Berl). 2003;170(1):94-101.16.

16. Torres-Lista V, Gimenez-Llort L. Early postnatal handling and environmental enrichment improve the behavioral responses of 17-month-old 3xTg-AD and non-transgenic mice in the Forced Swim Test in a gender-dependent manner. Behav Processes. 2015;120:120127.

17. Qi CC, Shu YM, Chen FH, Ding YQ, Zhou JN. Sensitivity during the forced swim test is a key factor in evaluating the antidepressant effects of abscisic acid in mice. Behav Brain Res. 2016;300:106-113.

18. Jacobson LH, Cryan JF. Feeling strained? Influence of genetic background on depression-related behavior in mice: a review. Behavior genetics. 2007;37(1):171-213.

19. Andreasen JT, Redrobe JP. Antidepressant-like effects of nicotine and mecamylamine in the mouse forced swim and tail suspension tests: role of strain, test and sex. Behav Pharmacol. 2009;20(3):286-295.

20. Kulikov AV, Tikhonova MA, Osipova DV, Kulikov VA, Popova NK Association between tryptophan hydroxylase-2 genotype and the antidepressant effect of citalopram and paroxetine on immobility time in the forced swim test in mice. Pharmacol Biochem Behav. 2011;99(4):683-687.

21. Osipova DV, Kulikov AV, Popova NK. C1473G polymorphism in mouse tph2 gene is linked to tryptophan hydroxylase-2 activity in the brain, intermale aggression, and depressive-like behavior in the forced swim test. J Neurosci Res. 2009;87(5):1168-1174.

22. Moloney RD, Dinan TG, Cryan JF. Strain-dependent variations in visceral sensitivity: relationship to stress, anxiety and spinal glutamate transporter expression. Genes Brain Behav. 2015;14(4):319-329.

23. Costa AP, Vieira C, Bohner LO, Silva CF, Santos EC, De Lima TC, et al. A proposal for refining the forced swim test in Swiss mice. Prog Neuropsychopharmacol Biol Psychiatry. 2013;45:150-155.

24. Li N, Xu Y, Chen X, Duan Q, Zhao M. Sex-specific diurnal immobility induced by forced swim test in wild type and clock gene deficient mice. Int J Mol Sci. 2015;16(4):6831-6841.

25. Pechnick RN, Chesnokova VM, Kariagina A, Price S, Bresee CJ, Poland RE. Reduced immobility in the forced swim test in mice with a targeted deletion of the leukemia inhibitory factor (LIF) gene. Neuropsychopharmacology. 2004;29(4):770-776.

26. Franceschelli A, Sens J, Herchick S, Thelen C, Pitychoutis PM. Sex differences in the rapid and the sustained antidepressant-like effects of ketamine in stress-naive and "depressed" mice exposed to chronic mild stress. Neuroscience. 2015;290:49-60.

27. Targowska-Duda KM, Feuerbach D, Biala G, Jozwiak K, Arias HR Antidepressant activity in mice elicited by 3-furan-2-yl-N-p-tolylacrylamide, a positive allosteric modulator of the alpha7 nicotinic acetylcholine receptor. Neurosci Lett. 2014;569:126-130.

28.Targowska-Duda KM, Jozwiak K, Arias HR. Role of the nicotinic receptor beta4 subunit in the antidepressant activity of novel N,6dimethyltricyclo[5.2.1.0(2),(6)]decan-2-amine enantiomers. Neurosci Lett. 2013;553:186-190.

29.Shoji H, Takao K, Hattori S, Miyakawa T. Age-related changes in behavior in C57BL/6J mice from young adulthood to middle age. Mol Brain. 2016;9:11.

30.Martin-Aragon S, Villar A, Benedi J. Age-dependent effects of esculetin on mood-related behavior and cognition from stressed mice are associated with restoring brain antioxidant status. Prog Neuropsychopharmacol Biol Psychiatry. 2016;65:1-16.

31. David DJ, Bourin M, Hascoet M, Colombel MC, Baker GB, Jolliet P. Comparison of antidepressant activity in 4- and 40-week-old male mice in the forced swimming test: involvement of 5-HT1A and 5-HT1B receptors in old mice. Psychopharmacology (Berl). 2001;153(4):443-449.

32. Yates G, Panksepp J, Ikemoto S, Nelson E, Conner R. Social isolation effects on the "behavioral despair" forced swimming test: effect of age and duration of testing. Physiol Behav. 1991;49(2):347-353.

33. Bogdanova OV, Kanekar S, D’Anci KE, Renshaw PF. Factors influencing behavior in the forced swim test. Physiology \& behavior. 2013;118:227239.

34.Veenema AH, Cremers TI, Jongsma ME, Steenbergen PJ, de Boer SF, Koolhaas JM. Differences in the effects of 5-HT(1A) receptor agonists on forced swimming behavior and brain 5-HT metabolism between low and high aggressive mice. Psychopharmacology (Berl). 2005;178(2-3):151-160.

35.Veenema AH, Meijer OC, de Kloet ER, Koolhaas JM. Genetic selection for coping style predicts stressor susceptibility. J Neuroendocrinol. 2003;15(3):256-267.

36. Kulkarni SK, Dhir A. Effect of various classes of antidepressants in behavioral paradigms of despair. Prog Neuropsychopharmacol Biol Psychiatry. 2007;31(6):1248-1254.

37. Frazer A. Pharmacology of antidepressants. J Clin Psychopharmacol. 1997;17 Suppl 1:2S-18S. 